\title{
Pump-Probe Measurements of Carrier Capture Times in Quantum Well Structures
}

\author{
Jian-Jang Huang, Ding-Wei Huang, Chung-Yen Chao, Jiun-Haw Li, and C. C. Yang \\ Institute of Electro-Optical Engineering and \\ Department of Electrical Engineering \\ National Taiwan University \\ Taipei, Taiwan, R. O. C. \\ (phone) 886-2-3635251 ext. 445 \\ (fax) 886-2-3652637 \\ (E-mail) ccy@.cc.ee.ntu.edu.tw
}

The captures of electrons and holes into semiconductor quantum well structures have received considerable attention in both fundamental and device-oriented research. In quantum well lasers, the capture efficiency is expected to influence both the quantum efficiency and dynamic performance. Two multiple quantum well (MQW) samples (waveguide structures) were used in our experiments. The first one consists of 55 quantum wells. The well and barrier thicknesses are $7 \mathrm{~nm}$ and $12 \mathrm{~nm}$, respectively. Because of the large barrier thickness, we called it as the uncoupled QW sample. The second one has 110 quantum wells. The well and barrier thicknesses are $3.5 \mathrm{~nm}$ and $5.5 \mathrm{~nm}$, respectively. It is actually a supperlattice and we called it as the coupled QW sample. In our experiments, we intend to estimate the carrier capture times with subpicosecond pump-probe measurements. The experimental setup is shown in Fig 1 . When the input laser pulse ( $80 \mathrm{fs}$ ) passes through the $14 \mathrm{~cm}$ fiber, the spectrum is broadened from $7 \mathrm{~nm}$ to about $100 \mathrm{~nm}$ (FWHM) due to self-phase modulation. We then use a grating in either pump or probe arm to select the required central wavelength and spectral width, and then re-compress the pulse down to $150 \mathrm{fs}$. With degenerate and nondegenerate pump-probe configurations, we can measure the decay time of carriers in the barrier states and the rise time of carriers in the confined subbands in both uncoupled and coupled quantum well structures. The temporal evolution of the decay of barrier carrier density in the uncoupled QW sample is shown in Fig. 2. The experiment was carried out at a pump laser power of $1.5 \mathrm{~mW}$, corresponding to an excitation carrier density of $3 \times 10^{16} \mathrm{~cm}^{-3}$ in the barrier states. The decay of the carrier density has two components. In the first $20 \mathrm{ps}$ (after the excitation of the pump pulse), the decrease of barrier density is slow; after that a sharp decrease occurs. Compared to the uncoupled sample, the decrease in the coupled quantum well structure has only one component which is quite sharp. In Fig. 3, the temporal evolution of the subband carrier density is shown. By fitting the experimental data to the numerical results based on a set of rate equations, we obtain a capture time of about 15 ps in the uncoupled QW structure and 8 ps in the coupled QW well structure. Actually, the slower decay 
of barrier carriers in the uncoupled sample, as shown in Fig. 2, confirms the slower rise of subband carriers, as shown in Fig. 3.

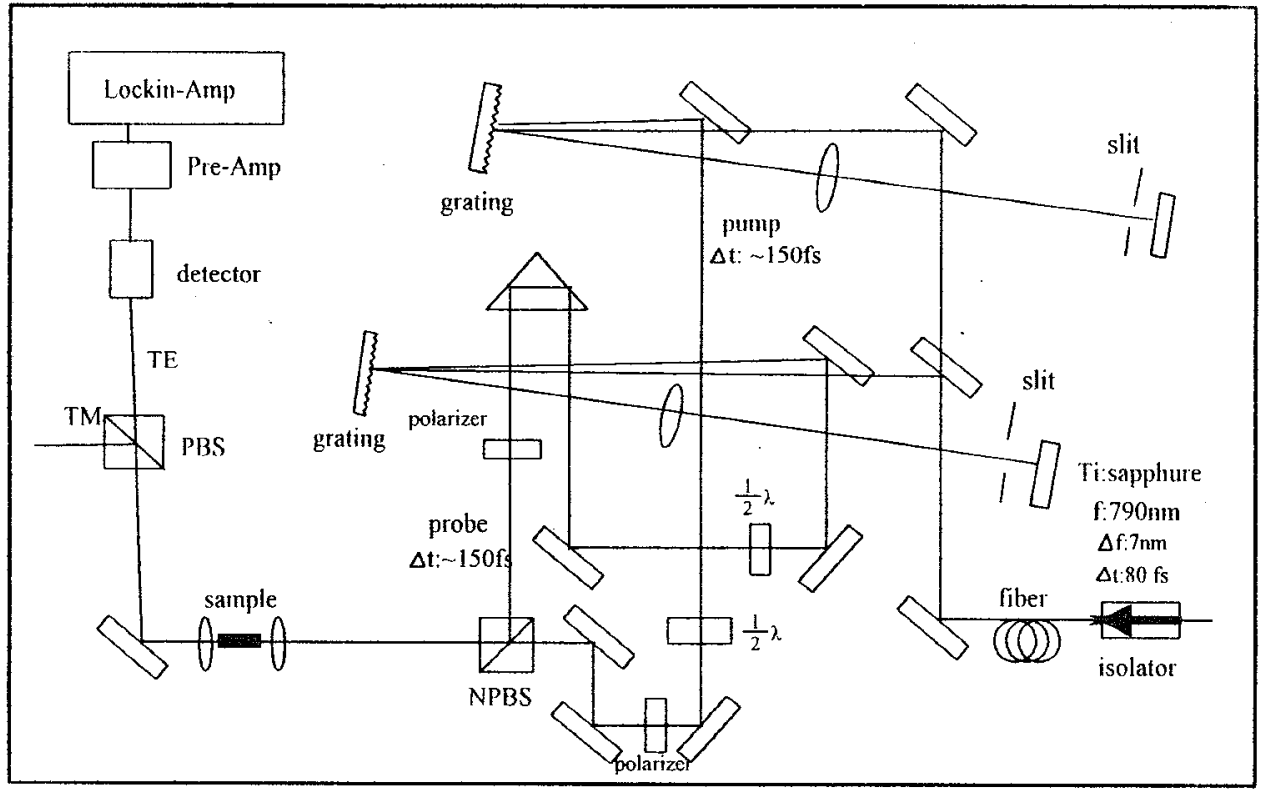

Fig. 1 Schematic diagram of the experimental setup.

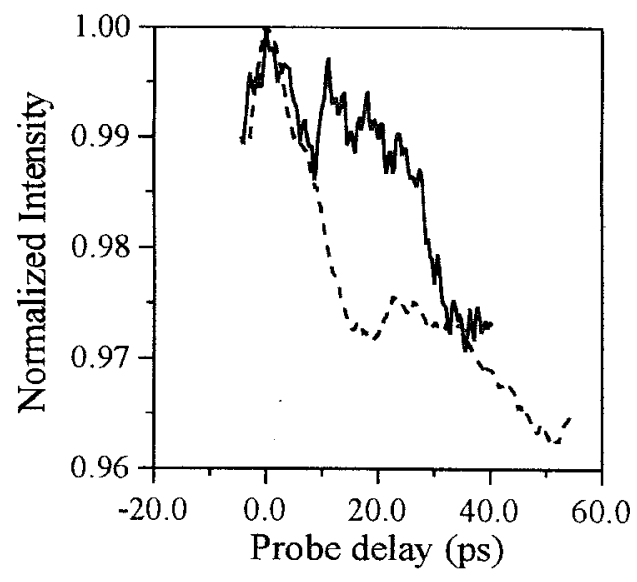

Fig. 2 Differential transmission in the degenerate experiments with uncoupled QW (solid curve) and coupled QW (dashed curve) samples.

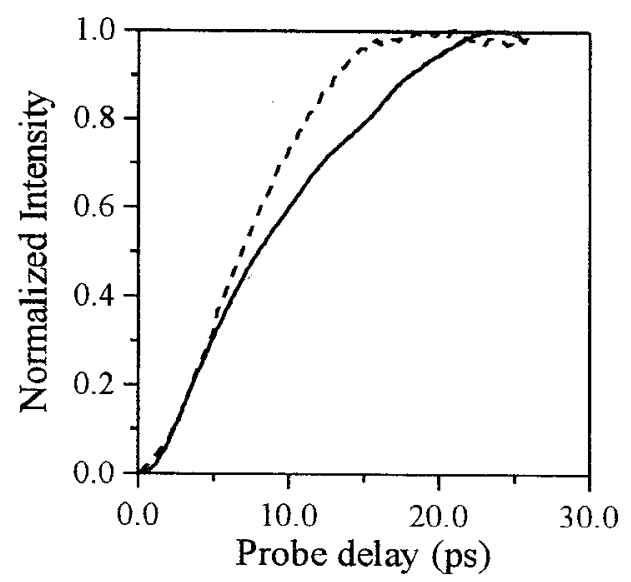

Fig. 3 Differential transmission in the nondegenerate experiments with uncoupled QW (solid curve) and coupled QW (dashed curve) samples. 